\title{
PIECEWISE LINEAR DISCONTINUOUS DOUBLE COVERINGS OF THE CIRCLE
}

\author{
ROZA GALEEVA AND CHARLES TRESSER
}

(Communicated by Charles Pugh)

\begin{abstract}
In his study of a particular Lorenz-like semiflow, S. F. Kennedy introduced a two-parameter family of endomorphisms of the circle with two marked points. These are piecewise affine double coverings of the circle with a pair of discontinuities, which all have topological entropy $\log 2$. We answer the question Kennedy raised about when two such maps are topologically conjugate.
\end{abstract}

\section{INTRODUCTION AND STATEMENT OF THE RESULTS}

In the wake of Williams's work on the Lorentz equations [7], Kennedy has described the semiflows on the branched 2-manifold $\mathbf{W}$ represented in Figure 1 (on the next page), where the sketch of phase portrait illustrates the main features of these semiflows [3]. Following backward the branches of the stable manifold of $O$, one gets two first intersection points at $P$ and $Q$ with the circle $\mathbf{C}$. The circle $\mathbf{C}$ with its two marked points is a natural section of this semiflow, so the topological dynamics of the semiflow can be captured by studying $K$-maps, i.e., double coverings of the circle with two marked points where the map can be discontinuous, and such that each arc between the marked points is sent to the full circle less one point. In this note, we shall restrict ourselves to the case of expanding $K$-maps with a constant factor 2 on the two arcs of continuity. Opening the circle at one marked point to form the interval $[0,1)$ with a marked point at $\frac{1}{2}$ means that we shall examine the two-parameter family $f_{(a, b)}:[0,1) \rightarrow[0,1)$, where

and

$$
\left.f_{(a, b)}\right|_{[0,1 / 2)}(x)=(2 x+a) \quad \bmod 1
$$

$$
\left.f_{(a, b)}\right|_{[1 / 2,1)}(x)=\left(2 x+b-\frac{1}{2}\right) \bmod 1 .
$$

For simplicity, we shall also denote by $\left\{f_{(a, b)}\right\}$ the corresponding family of $K$-maps. Following a question raised by Kennedy in $[3,4]$ we shall more precisely describe conditions on the parameters, which are necessary and sufficient for two $K$-maps in this family to be $K$-topologically conjugate, i.e., be the same up to a continuous change of coordinates, which preserves the set of marked points.

Received by the editors August 19, 1991.

1991 Mathematics Subject Classification. Primary 58F20, 58F14.

Key words and phrases. Kneading theory, topological conjugacy, branched manifolds. 


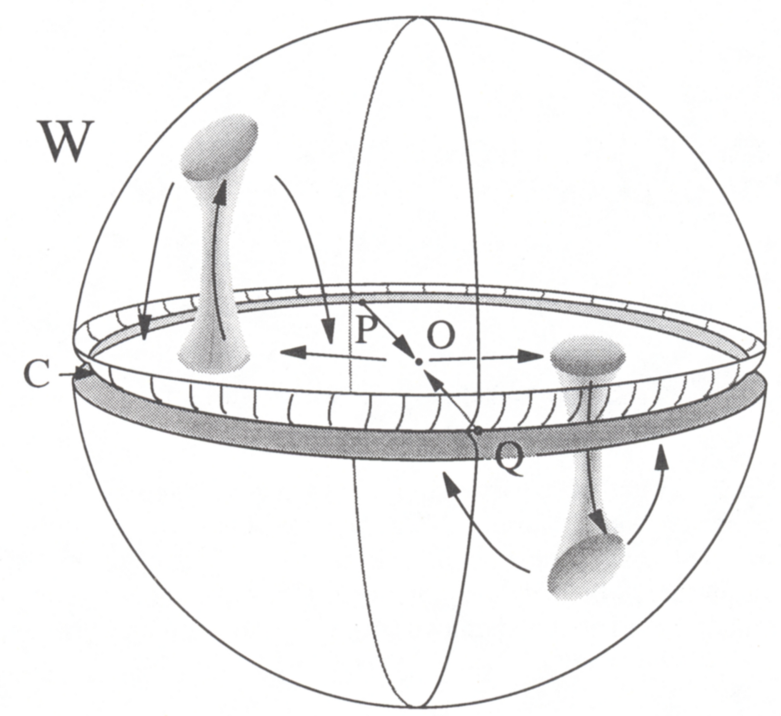

FIGURE 1

Remark. The topological conjugacy of semiflows on $\mathbf{W}$ of the kind described by Kennedy (see Figure 1 ) is equivalent to the $K$-topological conjugacy of the associated $K$-maps.

Often we will be switching from working with $K$-maps to working on the semi-open interval with one marked point, and vice versa, with no particular notice. With this freedom in switching between a space and a fundamental region of its covering space, the parameter space for the $K$-maps, which is the two-torus $\mathbf{T}^{2}=\mathbf{R}^{2} / \mathbf{Z}^{2}$, can equivalently be taken as (for instance) the set $\mathbf{S}=[0,1) \times[0,1)$; we are interested in exhibiting a subset $\mathbf{P}$ of $\mathbf{S}$, which represents all possible $K$-topological behavior, where no two points correspond to the same $K$-topological behavior of the circle map, and which is minimal in the sense that no subset of $\mathbf{P}$ represents all possible $K$-topological behavior. Call such a set a minimal parameter space. Our main result is the following:

Theorem 1. The convex set $\mathbf{K}$ defined as the union of the three regions $\left\{\frac{1}{4} \leq a<\right.$ $\left.\frac{1}{2}, \frac{1}{2}-a \leq b \leq a\right\},\left\{\frac{1}{2} \leq a \leq \frac{3}{4}, 0 \leq b \leq a\right\}$, and $\left\{\frac{3}{4}<a<1,0 \leq b<\frac{3}{2}-a\right\}$ $i$ a minimal parameter space for the family of $K$-maps $\left\{f_{(a, b)}\right\}$.

The region $\mathbf{K}$ is represented in Figure 2, where a heavy line on the boundary means that this line belongs to $\mathbf{K}$. The circled numbers in Figure 2 help to locate, in the parameter space, the $(a, b)$ pairs corresponding to the graphs represented in Figure 3. The pairs numbered 8 and 9 do not belong to $\mathbf{K}: 9$ is $K$-conjugate to 7 , and 8 to 2 .

Notice that the change of coordinate $x \rightarrow 1-x \quad K$-conjugates the maps $f_{(a, b)}$ and $f_{(1 / 2-b, 1 / 2-a)}$ and that on the circle identified with $[0,1] /-1 \sim 1$ the change of coordinate $x \rightarrow\left(x+\frac{1}{2}\right) \bmod 1 K$-conjugates the maps $f_{(a, b)}$ and $f_{(a, b)}$. This allows to replace $\mathbf{S}$ by the closure $\mathbf{K}_{1}$ of $\mathbf{K}$ without loosing any sort of dynamics up to $K$-topological conjugacy.

Remark. When $b=\left(a-\frac{1}{2}\right) \bmod 1, f_{(a, b)}$ has the same formula as an ordinary double covering map of the circle, and all these maps $f_{(a, b)}$ would represent 


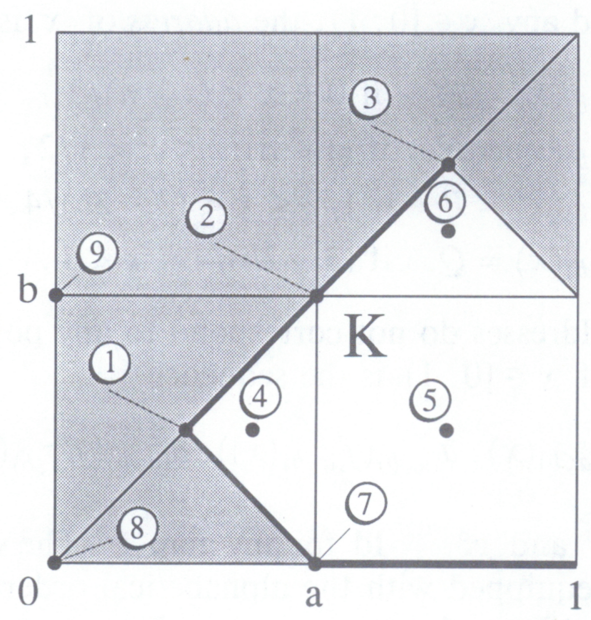

FIGURE 2

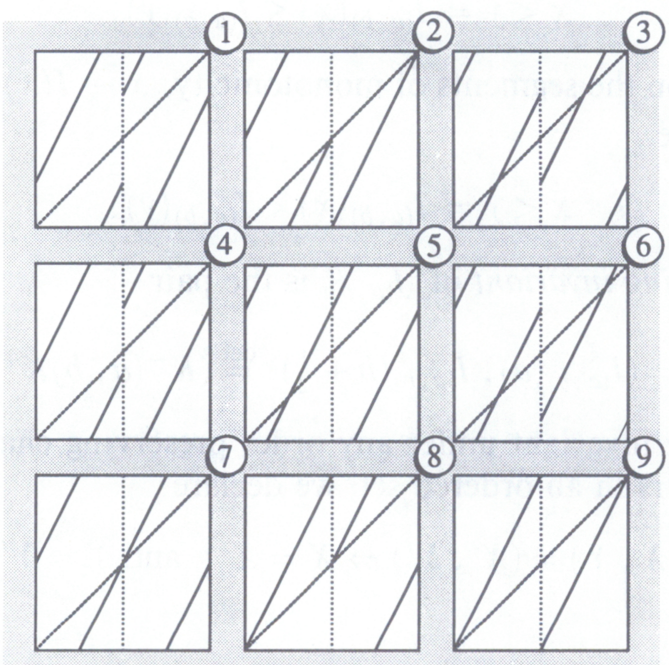

FIGURE 3

the same topological dynamics as double covers; however, these $f_{(a, b)}$ 's are not necessarily $K$-topologically conjugate (i.e., topologically conjugate as $K$-maps), and Theorem 1 tells us that indeed they are not $K$-conjugate.

Let us then mention that all maps with $b=\left(\frac{3}{2}-a\right) \bmod 1$ and $a>\frac{1}{2}$ form a single $K$-conjugacy class represented in $\mathbf{K}$ by the point $(a, b)=\left(\frac{3}{4}, \frac{3}{4}\right)$; for instance: for such parameter values, the circle splits into two arcs bounded by the images of 0 and $\frac{1}{2}$, where the map acts as $f(x)=2 x \bmod 1$ does on $[0,1)$. This allows us to reduce further the parameter space from $\mathbf{K}_{1}$ to $\mathbf{K}$, and it only remains to establish that no more reduction can be made.

This impossibility of further reduction will be proved using ideas from kneading theory [5], and we now recall what we need from this theory, in a form suitable for our purpose. Hence, for any map $f_{(a, b)}$ considered as a map on 
the interval $[0,1)$, and any $x \in[0,1)$, the address of $x$ is

$$
\begin{array}{ll}
\hat{a}_{(a, b)}(x)=M & \text { if } 0 \leq x<(1-a) / 2, \\
\hat{a}_{(a, b)}(x)=N & \text { if }(1-a) / 2 \leq x<1 / 2, \\
\hat{a}_{(a, b)}(x)=P & \text { if } 1 / 2 \leq x<(3-2 b) / 4, \\
\hat{a}_{(a, b)}(x)=Q & \text { if }(3-2 b) / 4 \leq x<1 .
\end{array}
$$

Notice that some addresses do not correspond to any point when $a=0$ or $b=0$. The itinerary of $x \in[0,1)$ is the sequence

$$
I_{(a, b)}(x)=\left(\hat{a}_{(a, b)}(x), \hat{a}_{(a, b)}\left(f_{(a, b)}(x)\right), \hat{a}_{(a, b)}\left(f_{(a, b)}^{\circ 2}(x)\right), \ldots\right),
$$

where $g^{\circ n}=g \circ g^{\circ(n-1)}$ and $g^{\circ 0} \stackrel{\text { def }}{=}$ Id for any map $g$. The set of infinite words in $\{M, N, P, Q\}$ is equipped with the alphabetical order on words induced by $M<N<P<Q$. Since the map $f_{(a, b)}$ is increasing on its segments of monotonicity, the map $x \mapsto I_{(a, b)}(x)$ is weakly order preserving, i.e.,

$$
x<y \Rightarrow I_{(a, b)}(x) \leq I_{(a, b)}(y) .
$$

Since $f_{(a, b)}^{\prime}=2$ on the segments of monotonicity, $x \mapsto I(x)$ is in fact strongly order preserving, i.e.,

$$
x<y \Rightarrow I_{(a, b)}(x)<I_{(a, b)}(y) .
$$

Then the kneading invariant of $f_{(a, b)}$ is the pair

$$
K(a, b)=\left(I_{(a, b)}(a), I_{(a, b)}\left(b+\frac{1}{2}\right)\right) \stackrel{\text { def }}{=}\left(K^{-}(a, b) K^{+}(a, b)\right),
$$

which is obviously invariant under any order preserving change of coordinate on $[0,1)$. For pairs in an ordered set, we declare

$$
(X, Y)=\left(X^{\prime}, Y^{\prime}\right) \Leftrightarrow X=X^{\prime} ; \text { and } Y=Y^{\prime}
$$

and

$$
(X, Y)>\left(X^{\prime}, Y^{\prime}\right) \Leftrightarrow X \geq X^{\prime} \text { and } Y>Y^{\prime} \text { or } X>X^{\prime} \text { and } Y \geq Y^{\prime} .
$$

From the above discussion on reductions of the parameter space due to simple changes of variables, Theorem 1 is a consequence of

Theorem 2. $K(a, b)=K\left(a^{\prime}, b^{\prime}\right)$ if and only if

- either $(a, b)=\left(a^{\prime}, b^{\prime}\right)$,

- or $b=\frac{3}{2}-a$ and $b^{\prime}=\frac{3}{2}-a^{\prime}$ with $\frac{1}{2}<a<1$ and $\frac{1}{2}<a^{\prime}<1$.

The next section is a proof of Theorem 2. This proof is elementary but definitely analytic. A main idea when studying parametrized families of maps is that the growth of the derivative with respect to the parameter is easier to control when there is some growth of the derivative with respect to the variable. After some successes for quadratic maps (see, e.g., [1]), simpler examples involving piecewise affine maps have been studied in $[6,2]$. Because the derivative $f_{(a, b)}^{\prime}(x)$ is always equal to 2 , our case is an even more straightforward example 
for the method, but we still do not know if a more topological approach could yield a proof of Theorems 1 and/or 2 .

\section{Proof of Theorem 2}

The parametrization of the family $\left\{f_{(a, b)}\right\}$ was natural in that it kept the symmetries of the original semiflow problem. We now change the scale to simplify the computations and, more importantly, the parametrization in such a way that proving Theorem 2 amounts to showing a strict monotonicity property of the kneading invariant. Hence we shall deal with the family $F_{(A, B)}:[-1,1[$ $\rightarrow[-1,1[$, with $(A, B) \in[-1,1) \times[-1,1)$ defined by

$$
F_{(A, B)}(x)= \begin{cases}2 x+A+2 & \text { for }-1 \leq x<(-A-1) / 2 \\ 2 x+A & \text { for }(-A-1) / 2 \leq x<0 \\ 2 x+B & \text { for } 0 \leq x<(1-B) / 2 \\ 2 x+B-2 & \text { for }(1-B) / 2 \leq x<1\end{cases}
$$

The relation between the two parametrizations is given by the formulas

$$
A=2 \cdot a-1, \quad B=2 \cdot\left[\left(\frac{1}{2}+b\right) \bmod 1\right]-1 .
$$

The kneading theory addresses for this family are defined like those for the family $\left\{f_{(a, b)}\right\}$. A main virtue of the new parametrization is that

$$
(A, B)<\left(A^{\prime}, B^{\prime}\right) \Rightarrow\left(F_{(A, B)}(-1), F_{(A, B)}(0)\right)<\left(F_{\left(A^{\prime}, B^{\prime}\right)}(-1), F_{\left(A^{\prime}, B^{\prime}\right)}(0)\right)
$$

and

$$
(A, B)<\left(A^{\prime}, B^{\prime}\right) \text { and } x<y \Rightarrow I_{(A, B)}(x)<I_{\left(A^{\prime}, B^{\prime}\right)}(y) .
$$

It is then clear that Theorem 2 is equivalent to

Theorem $2^{\prime}$. $K(A, B)=K\left(A^{\prime}, B^{\prime}\right)$ if and only if

- either $(A, B)=\left(A^{\prime}, B^{\prime}\right)$,

- or $B=-A$ and $B^{\prime}=-A^{\prime}$ with $0<A<1$ and $0<A^{\prime}<1$.

Set

$$
x_{n}=F_{(A, B)}^{n}(A), \quad y_{n}=F_{(A, B)}^{n}(B)
$$

and

$$
s_{n}=\partial x_{n} / \partial A, \quad t_{n}=\partial x_{n} / \partial B, \quad u_{n}=\partial y_{n} / \partial A, \quad v_{n}=\partial y_{n} / \partial B
$$

We shall say that the map $F_{(A, B)}$ (or the pair $(A, B)$ ) is $A$-isolated if $x_{n}<0$ for all $n \geq 0$. Similarly the map $F_{(A, B)}$ (or the pair $(A, B)$ ) is $B$-isolated if $y_{n}>0$ for all $n \geq 0$.

Lemma 1. $\forall n>0: s_{n}>0, t_{n} \geq 0, u_{n} \geq 0, v_{n}>0$.

Furthermore,

- $\forall(A, B)$ not $A$-isolated, $\exists M_{(A, B)}$ finite such that $t_{n}>0$ for $n>M_{(A, B)}$ and $\lim _{n \rightarrow \infty} t_{n}=\infty$,

- $\forall(A, B)$ not $B$-isolated, $\exists N_{(A, B)}$ finite such that $u_{n}>0$ for $n>N_{(A, B)}$ and $\lim _{n \rightarrow \infty} u_{n}=\infty$,

- $\forall A$ and $\forall B, \lim _{n \rightarrow \infty} s_{n}=\lim _{n \rightarrow \infty} v_{n}=\infty$. 
Proof of Lemma 1. We shall only examine the cases of $s_{n}$ and $t_{n}$-the other two cases being similar. A direct computation yields

$$
s_{n+1}=\left\{\begin{array}{ll}
2 \cdot s_{n}+1 & \text { for }-1 \leq x_{n}<0, \\
2 \cdot s_{n} & \text { for } 0 \leq x_{n}<1,
\end{array} \quad \text { with } s_{0}=1\right.
$$

and

$$
t_{n+1}=\left\{\begin{array}{ll}
2 \cdot t_{n} & \text { for }-1 \leq x_{n}<0, \\
2 \cdot t_{n}+1 & \text { for } 0 \leq x_{n}<1,
\end{array} \text { with } t_{0}=0 .\right.
$$

Lemma 1 then follows from these formulas and from the definitions of $A$ and $B$-isolated.

Proposition 1. For any pair $(A, B)$,

$$
\left(A^{\prime}, B^{\prime}\right)>(A, B) \Rightarrow K\left(A^{\prime}, B^{\prime}\right)>K(A, B) \text {. }
$$

Furthermore, if $(A, B)$ is $A$ or $B$-isolated then

$$
\left(A^{\prime}, B^{\prime}\right)=(A, B) \Leftrightarrow K\left(A^{\prime}, B^{\prime}\right)=K(A, B) .
$$

Proof of Proposition 1. If $(A, B)$ is neither $A$ nor $B$-isolated, Proposition 1 follows immediately from Lemma 1 . If, for instance, $(A, B)$ is $A$-isolated, then $K^{-}$depends on $A$ but not on $B$, and there are two cases to consider, i.e., $(A, B)$ is either $B$-isolated or not. However, in both cases, $K^{+}$depends on $B$, which is enough to prove the equivalence stated in the proposition.

Following the method used in [2], we shall next investigate how $K^{-}$and $\mathrm{K}^{+}$ depend on $C=A+B$ and $D=A-B$. To this end, we set

$$
S_{n}=\partial x_{n} / \partial C, \quad T_{n}=\partial x_{n} / \partial D, \quad U_{n}=\partial y_{n} / \partial C, \quad V_{n}=\partial y_{n} / \partial D
$$

We shall say that the map $F_{(A, B)}$ (or the pair $(A, B)$ ) is A-prefixed if $B \leq 0$ and $F_{(A, B)}(A)$ is the unique fixed point of $F_{(A, B)}$ greater than zero. Similarly the map $F_{(A, B)}$ (or the pair $(A, B)$ ) is B-prefixed if $A>0$ and $F_{(A, B)}(B)$ is the unique fixed point $F_{(A, B)}$ smaller than zero.

Lemma 2. $\forall n>0: S_{n}>2, T_{n} \geq 1, U_{n}>2, V_{n} \leq-1$.

Furthermore,

- for $(A, B)$ not A-prefixed, $\exists M_{(A, B)}^{\prime}$ finite such that $T_{n}>2$ for $n>$ $M_{(A, B)}^{\prime}$ and $\lim _{n \rightarrow \infty} T_{n}=\infty$,

- for $(A, B)$ not B-prefixed, $\exists N_{(A, B)}^{\prime}$ finite such that $V_{n}<-2$ for $\left.n\right\rangle$ $N_{(A, B)}^{\prime}$ and $\lim _{n \rightarrow \infty}|V|_{n}=\infty$,

- $\forall A$ and $\forall B, \lim _{n \rightarrow \infty} S_{n}=\lim _{n \rightarrow \infty} U_{n}=\infty$.

Proof of Lemma 2. Similar to the proof of Lemma 1, this proof follows from the definitions and a direct computation, which gives us the following recursion relations:

$$
\begin{aligned}
& S_{n+1}=2 \cdot S_{n}+1 \quad \text { for }-1 \leq x_{n}<1, \quad \text { with } S_{0}=1 \text {; } \\
& T_{n+1}=\left\{\begin{array}{ll}
2 \cdot T_{n}+1 & \text { for }-1 \leq x_{n}<0, \\
2 \cdot T_{n}-1 & \text { for } 0 \leq x_{n}<1,
\end{array} \text { with } T_{0}=1 ;\right. \\
& U_{n+1}=2 \cdot U_{n}+1 \text { for }-1 \leq x_{n}<1, \quad \text { with } U_{0}=1 \text {; } \\
& V_{n+1}=\left\{\begin{array}{ll}
2 \cdot V_{n}+1 & \text { for }-1 \leq x_{n}<0, \\
2 \cdot V_{n}-1 & \text { for } 0 \leq x_{n}<1,
\end{array} \quad \text { with } V_{0}=-1 .\right.
\end{aligned}
$$

The maps which are either $A$-prefixed or $B$-prefixed, but not both, offer no particular difficulty, so Lemma 2 implies the following 
Proposition 2. Assume $F_{(A, B)}$ is not both A-prefixed and B-prefixed. Then, writing $C=A+B, D=A-B$ and $C^{\prime}=A^{\prime}+B^{\prime}, D^{\prime}=A^{\prime}-B^{\prime}$, we have

$$
\left(C^{\prime}, D^{\prime}\right)>(C, D) \Rightarrow K^{-}\left(A^{\prime}, B^{\prime}\right)>K^{-}(A, B)
$$

and

$$
\left(C^{\prime},-D^{\prime}\right)>(C,-D) \Rightarrow K^{+}\left(A^{\prime}, B^{\prime}\right)>K^{+}(A, B) .
$$

Two pairs $(A, B) \neq\left(A^{\prime}, B^{\prime}\right)$, such that one at least is not both $A$-prefixed and $B$-prefixed, can always be compared using Propositions 1 and 2, with the conclusion that $K(A, B) \neq K\left(A^{\prime}, B^{\prime}\right)$. If both pairs are both $A$-prefixed and $B$-prefixed, and $A \cdot(1+A) \cdot A^{\prime} \cdot\left(1+A^{\prime}\right) \neq 0$ the two maps represent the same dynamics, as already mentioned. At last the $A$ - and $B$-prefixed cases $A=-1$ and $A=0$ are different from each other and from anything else.

\section{ACKNOWLEDGMENTS}

We heard about this problem from many sources and would like to thank particularly C. Sparrow for first telling us about it, as well as K. Brucks and $\mathrm{S}$. Kennedy for recalling it to our attention. This work was made possible by the second U.S.A.-U.S.S.R. meeting on "Chaos" held in Tarusa (U.S.S.R), in August 1990.

\section{REFERENCES}

1. M. Benedicks and L. Carleson, On iterations of $1-a x^{2}$ on $(-1,1)$, Ann. of Math. (2) 122 (1985), 1-25.

2. R. Galeeva, Kneading sequences of piecewise bimodal maps, Chaos (to appear).

3. S. F. Kennedy, $A$ Lorenz-like strange attractor, Ph.D. thesis, Northwestern University, 1988.

4. _ The topology of the periodic points set of a family of circle maps, preprint, Univ. of Delaware, 1989.

5. J. Milnor and W. Thurston, On iterated maps of the interval, Lecture Notes in Math., vol. 1342, Springer-Verlag, New York, 1988, pp. 465-563.

6. M. Misiurewicz and E. Visinescu, Kneading sequences of skew tent maps, Ann. Inst. H. Poincaré 27 (1991), 125-140.

7. R. F. Williams, The structure of Lorenz attractors, Inst. Hautes Études Sci. Publ. Math. $\mathbf{5 0}$ (1979), 321-347.

Joint Institute for Nuclear Research, LCTA, P.O. Box 79, Moscow 101000, Republic oF Russia

E-mail address: GALEEVA@MAINZ.JINR.DUBNA.SU

I.B.M., T. J. Watson Research Center, P.O. Box 218, Yorktown Heights, New York 10598

E-mail address:TRESSER@YKTVMV.BITNET 